\title{
Influence of Ageing and Educational Level on the Prevalence of Body-Part- as-Objects in Normal Subjects
}

\author{
Philippe Peigneux ${ }^{(1)}$, Martial van der Linden ${ }^{(2)}$ \\ (1) Centre de Recherches du Cyclotron, Service de Neurologie, Université de Liège, Belgium \\ (2) Centre de Recherches du Cyclotron, Service de Neuropsychologie, Université de Liège, Belgium
}

\begin{abstract}
When pantomiming gestures to verbal command, the overall production of body-part-as-object (BPO) errors is differentially affected by age and educational level in normal subjects. The higher overall frequency of BPO with age may be related to difficulties in the inhibition of the automatic activation of tool emblems (i.e., the BPO) instead of the appropriate hand posture to hold the tool. However, all subjects are equally able to correct errors in BPO on subsequent trials, which suggests the integrity of these gestural representations. In a clinical perspective, BPO data have therefore to be considered carefully when one wants to include this error type as an indicator of limb apraxia.
\end{abstract}

When asked to pantomime transitive gestures to verbal command, patients suffering from limb apraxia make different kinds of errors which may reflect specific disruptions in the conceptual or temporal-spatial stages implied in the processing of learned gestures (Rothi \& Heilman, 1997). To use a body part to represent the imagined object (e.g., to stick the index and middle finger straight out to represent the blades when required to pantomime the use of scissors) is a response considered to be commonly associated with apraxia, and was initially described by Goodglass and Kaplan (1963) as a "body-part-as-object" (BPO) error. They hypothesized that the production of a BPO error is an attempt to reduce the difficulty of the pantomime task by providing a more concrete representation of the tool. In an alternative view, Raymer, Maher, Foundas, Heilman, and Rothi (1997) suggest that what they call "body-part-as-tool" (BPT) errors are committed because there is an impairment in the ability to portray the correct hand posture used to hold a tool and instead the patient portrays the tool itself.

The assertion that these BPO errors have a pathological status and are indicators of limb apraxia is still subject to debate. Studies focusing on the differences in the production of BPO errors in normal and brain-damaged subjects have raised contradictory views as to the specificity of the BPO errors in association with the apraxic symptomatology (Haaland \& Flaherty, 1984; Poeck, 1986; Rothi, Mack, Verfaellie, Brown, \& Heilman, 1988) or, on the contrary, to their unspecificity from a pathological point of view because these errors are also commonly observed in normal subjects, although not at the same rate (Duffy \& Duffy, 1989; McDonald, Tate \& Rigby, 1994; Mozaz, Pena, Barraquer \& Marti, 1993). Raising an interesting methodological point, a recent study (Raymer et al., 1997) showed that BPO responses can nevertheless be considered as apraxic errors when subjects persevere in their error after having been warned to perform carefully the gesture "as if they really held the tool" for the second trial. They demonstrated that even if normal subjects indeed exhibit BPO responses when asked to pantomime transitive gestures to verbal command, they usually correct this error on the second trial, which is not the case for patients suffering from limb apraxia.

However, it could be the case that non-pathologic general factors could affect the production of BPO errors when pantomiming transitive gestures. Ska and Nespoulos (1987) have already shown that BPO is a more frequent behaviour when performed by aged subjects in pantomiming. Yet, they did not control for BPO corrections, which leaves the question if ageing influences the production of so-called "true" uncorrected BPO errors. In the present study, we focused on the possible influence of age on the production of BPO errors and on their correction in a normal population. Additionally, we investigated the influence of educational level on the occurrence of these BPO errors in elderly subjects. If these factors are shown to be related to the production of BPO errors in normal subjects, then these variables have to be taken into account as well in the evaluation of the performances of apraxic patients.

\section{METHOD}

\section{Subjects}

One hundred and sixty healthy right-handed volunteers participated in this experiment. None of them reported any psychiatric or neurological disability such as, for example, stroke, epilepsy, head injury, cerebral vascular 
disease, thromboses, or movement disorders. Three groups were established on the basis of two main criteria: age (Young or Old) and level of education (Low or High). A low level of education referred to a maximum of 8 years of schooling, and a high level to a minimum of 12 years of schooling. The Young-High (YH) group comprised 28 women and 27 men with a mean age of 23.1 years (range $18.3-30.2$ years, $S E M=0.34$ ) and a high level of education with a mean school attendance of 14.4 years (range $12-19$ years. $S E M=0.21$ ). The OldHigh $(\mathrm{OH})$ group included 26 women and 26 men with a mean age of 65.4 years (range $58.9-72.7$ years. SEM $=0.55)$

and a high level of education with a mean school attendance of 13.8 years (range $12-19$ years. $S E M=0.32$ ). The Old-Low (OL) group comprised 27 women and 26 men with a mean age of 68.6 years (range $58.7-75.9$ years. $S E M=0.67$ ) and a low level of education with a mean school attendance of 7.2 years (range $4-9$ years. $S E M=0.17$ ). A fourth group of young subjects with a low level of education was actually not constituted because such a low level of schooling ( 8 years) is lower than the actual Belgian compulsory schooling limit. Subsequently, this educational level in young adults was too likely to be associated with social or psychological factors, although it is a normal level of education for older adults. Preliminary analyses showed that the $\mathrm{OH}$ and YH groups did not differ significantly for educational level, $t(105)=1.55, p>.1$, but that $\mathrm{OH}$ and OL groups differed for age, $t(103)=-3.67, p<.001$.

\section{Experimental task}

All participants were verbally asked to pantomime 75 transitive gestures selected from previously reported experiments (Duffy et al., 1989; Mozaz et al., 1993; Raymer et al., 1997) and from the ULg (University of Liege) Praxis Assessment Battery (Peigneux \& Van der Linden, 1998). They were instructed as follows: "Now you are to pantomime common gestures of object use. It is important to note that you have to pantomime these gestures exactly as if you really held the object in your hand and used it. For example (examiner demonstration), if I ask you to pantomime how to unscrew a light bulb, it is important apart from the twisting movement itself, to leave a space between your fingers for the light bulb, and you must not make a fist to represent this light bulb'. After each incorrect BPO response, the examiner reinstructed systematically the subject to " ... pantomime the gesture exactly as if you actually used the tool " without further details and a second trial was allowed, otherwise the next item was initiated.

\section{Scoring and analysis}

Each participant's BPO response was recorded separately for each pantomime and for each trial by the examiner. All BPO errors were coded as "simple" or "consistent" BPO as follows. A simple BPO was considered to be an error that occurred on the first trial and which was accurately corrected after reinstruction. A consistent BPO was an error that occurred on the two trials despite the reinstruction provided by the examiner. This distinction was drawn in order to account for previous findings (Raymer et al., 1997) suggesting that only consistent BPOs are pathological responses. Four measures of performance were computed for each participant: (1) the sum of simple BPOs; (2) the sum of consistent BPOs; (3) the overall occurrence of BPOs (i.e., equal to (1)+(2)), representing the number of BPOs elicited at the first trial, and (4) the proportion of BPO errors accurately corrected after reinstruction (i.e., equal to (1)/(3)), which give us information on the subject's capacity to evoke the appropriate hand posture after having produced a BPO error. The ageing effect on these variables was assessed by comparing performances between younger and older subjects with the same high level of education (OH vs. YH), and educational level effect was assessed comparing performances between older subjects with a low and a high level of education (OL vs. $\mathrm{OH})$. The statistical non parametric test for comparisons between independent samples was the Mann-Whitney test, with a level of significance set at $p<.05$.

\section{RESULTS}

Simple BPO errors were reported in the YH $(M=1.18, S E M=0.14), \mathrm{OH}(M=2.04, S E M=0.26)$, and OL $(M=$ 3.26, $S E M=0.30$ ) groups. The Mann-Whitney test disclosed a statistically significant effect of the age factor on the production of simple $\mathrm{BPO}$ errors, $\mathrm{YH}<\mathrm{OH}, \mathrm{Z}$ adj. $=-2.25, p<.05$, as well as of the educational level factor, $\mathrm{OH}<\mathrm{OL}, \mathrm{Z}$ adj. $=-3.07, p<.005$.

Consistent BPO errors were also reported in the YH $(M=0.35, S E M=0.14), \mathrm{OH}(M=0.56, S E M=0.20)$, and OL $(M=1.40, S E M=0.33)$ groups. The Mann-Whitney test failed to show any significant age-related differences on the production of consistent $\mathrm{BPO}$ errors, $\mathrm{YH}=\mathrm{OH}, \mathrm{Z}$ adjusted $=-.32, p>.70$, but revealed a statistically significant difference related to the educational level effect, $\mathrm{OH}<\mathrm{OL}, \mathrm{Z}$ adjusted $=-2.34, p<.05$. The follow-up analysis confirms the lack of differences between the $\mathrm{YH}$ and $\mathrm{OH}$ groups, showing that the age effect (YH vs. OH) yielded a very small effect size $(-0.15)$ and that a sample size of 698 would be needed to reach a power level of .80 .

For the overall occurrence of BPO errors in the YH $(M=1.53, S E M=0.20), \mathrm{OH}(M=2.60, S E M=0.36)$, and OL $(M=4.66, S E M=0.39)$ groups, Mann-Whitney tests disclosed both significant effects of age, $\mathrm{YH}<\mathrm{OH}, \mathrm{Z}$ 
adj. $=-2.06, p<.05$, and of educational level, $\mathrm{OH}<\mathrm{OL}, \mathrm{Z}$ adj. $=-4.1, p<.001$. Actually, this result is similar to that reported for the production of simple BPO, but the simple BPO represents $74 \%$ of the overall occurrence of BPO errors, which explains those similarities.

Finally, the proportion of BPO errors accurately corrected after reinstruction in the YH $(M=86 \%, S E M=0.04)$, $\mathrm{OH}(M=87 \%, S E M=0.04)$, and OL $(M=79 \%, S E M=0.04)$ groups did not differ significantly with age, $\mathrm{OH}=$ $\mathrm{OL}, \mathrm{Z}$ adj. $=-1.62, p>.1$, nor educational level, $\mathrm{YH}=\mathrm{OH}, \mathrm{Z}$ adj. $=-0.17, p>.85$. Follow-up power analysis evidenced that age (YH vs. OH) yielded very small effect size $(-0.02)$ and that a sample size of 41,020 would be needed in order to reach a power level of .80, whereas for educational level (OH vs. OL), effect size was small (0.26 ) and a sample size of 226 was needed to reach the power level above.

The 75 transitive gestures proposed to each subject were analyzed in order to determine the susceptibility of specific items to produce BPO errors. Among the 160 subjects, 122 consistent BPO and 344 simple BPO errors were recorded by the examiner (see details in Appendix). Some gestures were found to be particularly associated with the production of BPO responses: "use scissors to cut paper"' (15.2\% of the overall BPO errors), "fire a revolver" (9.2\% BPO), "seal a document" and "look through binoculars" (6.0\% BPO each), and "draw a square with a pencil”" (5.4\% BPO). Seven other gestures elicited from $2.2 \%$ to $4.1 \%$ BPO responses, 43 gestures induced less than 2\% BPO responses among all the subjects, and 20 gestures did not elicit any BPO at all.

\section{DISCUSSION}

In the present study, we showed a significant effect of ageing and educational level on the production of simple $\mathrm{BPO}$ errors (i.e., errors accurately corrected at the second trial after reinstruction) as well as on the overall production of BPO errors. Nevertheless, the production of consistent BPO errors (not corrected at the second trial) and the ratio of BPO errors accurately corrected did not differ significantly between younger and older normal subjects.

The report of an influence of ageing on the production of simple or overall BPO errors is in line with a previous study (Ska \& Nespoulos, 1987) showing that aged subjects' performance differs from younger subjects' performance mainly in the static constituents of unimanual pantomimes, with a more frequent occurrence of BPO errors in the aged subjects. Also consistent is the report of BPO errors in normal elderly subjects compared with matched brain-damaged patients (e.g., Duffy \& Duffy, 1989; McDonald, Tate, \& Rigby, 1994). To account for the phenomena of BPO production, it was suggested that the BPO "materializes" the object and therefore may be an attempt to evade the difficulties of reproducing a transitive gesture outside of its concrete context (Goodglass \& Kaplan, 1963); but it may also reflect the inability to access the representation of the appropriate hand-posture (Raymer et al., 1997) or a poor representation of the external object (Haaland \& Flaherty, 1984). It was reported however that normal subjects are able to correct their error in a second trial whereas apraxic patients were not, and therefore that only consistent BPO errors can be considered as truly pathologic errors (Raymer et al., 1997). In our study, younger and older subjects did not differ for the prevalence of consistent $\mathrm{BPO}$ errors or for the proportion of corrected BPO, which suggests that the ability to call to mind the correct hand posture used to hold the imaginary tool is not affected per se by ageing, with all subjects being equally able to correct their BPO error after careful reinstruc-tion. It should be noted that the prevalence of these consistent $\mathrm{BPO}$ errors is very low in our $\mathrm{OH}$ and $\mathrm{YH}$ groups, with a global mean percentage of $0.6 \%$ of consistent BPO errors produced on the 75 required pantomimes.

Furthermore, Raymer et al. (1997) pointed out that in the various studies concerning BPO, researchers rarely clarify the nature of the instructions given to subjects. If normal and braindamaged subjects were compared in these studies on the global prevalence of simple BPO errors, then it could be that the age of the control population could potentially account as a confounding factor for the results described in these studies, in addition to a lack of explicit reinstruction after production of a BPO error. Moreover, our item analysis showed that the vulnerability of the different gestures to the elicitation of BPO responses is highly variable, with some gestures inducing easily this kind of response, and others not at all, as showed in the Appendix and already pointed out by Duffy and Duffy (1989). The kind of gestures selected for testing and the kind of reinstruction could thus lead to different results and this has to be taken into account when assessing patients for limb apraxia.

Finally, it was suggested (Raymer et al., 1997) that the individuals recall culturally acceptable emblem gestures when asked to perform some transitive gestures. We suggest that these emblematics, which have a strong symbolic representation, are automatically activated when subjects are required to pantomime the associated gesture and that, instead of a difficulty to evoke the correct hand posture used to hold the tool, it might be that the greatest difficulty in this case is to inhibit the activation of this automatic gesture. Ageing is known to be associated with inhibitory decline (Van der Linden et al., in press; Zacks \& Hasher, 1994), and our results show that the Old group committed more simple BPO errors than the Young matched group, a result in accordance with the hypothesis that ageing affects the ability to inhibit the associated emblematic response when required to pantomime a gesture, but did not affect the correct representation of the gesture per se. Indeed, elderly subjects demonstrated relative to younger subjects a similar ability to evoke the appropriate posture when reinstructed, 
because their attention was drawn to their error.

Regarding the educational level effect, our study disclosed a significant difference between higher and lower educated subjects for the production of simple as well as consistent BPO errors. Does it mean that a low level of education is associated with a greater tendency to create a context for tool use or with greater difficulties to evoke the appropriate hand-shaping representation? Actually, it is difficult to answer this question because this difference was only assessed in aged subjects, due to the practical difficulties to constitute a group of younger subjects with a low level of education. However, it must be noted that the $\mathrm{OH}$ and OL groups did not actually differ when compared for the proportion of BPO errors accurately corrected after reinstruction. This suggests that even if lower educated normal subjects produced more simple and consistent BPO errors than higher educated subjects, they correct their BPO errors at the same rate. It is therefore likely that the significant difference for consistent BPO production is simply a consequence of a largely higher overall occurrence of BPO in lower educated aged subjects, and therefore we cannot conclude that lower educated aged subjects are more impaired in their ability to correct their BPO errors than younger subjects. Further studies will be necessary to clarify the nature of the increased occurrence of BPO errors in lower educated subjects, but the noticeable fact that the level of education was shown to be associated with the prevalence of simple and consistent BPO errors in normal aged subjects implies that it must be considered carefully when one wants to include these errors as an indicator of a pathological apraxic status. In this respect, the proportion of corrected errors seems to be a more valid measure in this case, because this measure is independent of the overall frequency of errors. Moreover, Raymer et al. (1997), using a similar proportional measure, have disclosed a significant difference between left brain-damaged patients and matched controls in their ability to modify a BPO error after reinstruction, which suggests its validity in differentiating normal and pathological BPO behaviours.

\section{REFERENCES}

[1] Duffy, R.J., \& Duffy, J.R. (1989). An investigation of body part as object (BPO) responses in normal and brain-damaged adults. Brain and Cognition, 10, 220-236.

[2] Goodglass, H., \& Kaplan, E. (1963). Disturbance of gesture and pantomime in aphasia. Brain, 86, 703-720.

[3] Haaland, K.Y., \& Flaherty, D. (1984). The different types of limb apraxia errors made by patients with left vs. right hemisphere damage. Brain and Cognition, 3, 370-384.

[4] McDonald, S., Tate, R.L., \& Rigby, J. (1994). Error types in ideomotor apraxia: A qualitative analysis. Brain and Cognition, 25, 250270 .

[5] Mozaz, M.J., Pena, J., Barraquer, L.L., \& Marti, J. (1993). Use of body part as object in brain-damaged subjects. Third IBRO World Congress of Neuroscience (1991, Montreal, Canada). The Clinical Neuropsychologist, 7, 39-47.

[6] Peigneux, P., \& Van der Linden, M. (1998). Evaluation des Praxies théoriquement fondée: La B.E.P. Revue Neurologique, 154(Suppl. 1), 99-99.

[7] Poeck, K. (1986). The clinical examination for motor apraxia. Special Issue: Methods in neuropsychology. Neuropsychologia, 24, 129134.

[8] Raymer, A.M., Maher, L.M., Foundas, A.L., Heilman, K.M., \& Rothi, L.J. (1997). The significance of body part as tool errors in limb apraxia. Brain and Cognition, 34, 287-292.

[9] Rothi, L.G., \& Heilman, K.M. (1997). Apraxia: The cognitive neuropsychology of action. Hove, UK: Psychology Press.

[10] Rothi, L.J.G., Mack, L., Verfaellie, M., Brown, P., \& Heilman, K.M. (1988). Ideomotor apraxia: Error pattern analysis. Aphasiology, 2, 381-388.

[11] Ska, B., \& Nespoulous, J.L. (1987). Pantomimes and aging. Journal of Clinical \& Experimental Neuropsychology, 9, 754-766.

[12] Van der Linden, M., Hupet, M., Feyereisen, P., Schelstraete, M.A., Bestgen, Y., Bruyer, R., Lories, G., El Ahmadi, A., \& Seron, X. (in press). Cognitive mediators of age-related differences in language comprehension and verbal memory performance. Aging, Neuropsychology, and Cognition.

[13] Zacks, R.T., \& Hasher, L. (1994). Directed ignoring: Inhibitory regulation of working memory. In D. Dagenbach \& T.H. Carr (Eds.), Inhibitory processes in attention, memory, and language (pp. 241-264). San Diego, CA: Academic Press. 


\section{APPENDIX}

List of the 75 objects verbally presented for pantomime use, with the numbers of BPO errors elicited for each item across the 160 subjects as follows: the overall occurrence of BPO errors (column " $\mathrm{O}$ ', i.e., the number of subjects who made a BPO error for this item), and the number of consistent BPO errors (column " $\mathrm{C}$ "; i.e., the number of subjects who commit a BPO error for this item, and cannot accurately correct it after being reinstructed).

\begin{tabular}{|c|c|c|c|c|c|}
\hline ITEM & $\mathrm{O}$ & $\mathrm{C}$ & ITEM & $\mathrm{O}$ & $\mathrm{C}$ \\
\hline Scissors & 71 & 12 & Paintbrush (to paint a wall with) & 2 & 1 \\
\hline Revolver & 43 & 9 & Shovel & 2 & 1 \\
\hline Seal (a document) & 28 & 9 & Hammer & 2 & 0 \\
\hline Binoculars & 28 & 5 & Switch & 2 & 0 \\
\hline Pencil (draw a square with) & 25 & 2 & Adjustable wrench & 2 & 0 \\
\hline Lipstick (put on) & 19 & 6 & Saltshaker & 2 & 0 \\
\hline Phone (to answer) & 18 & 4 & Reel (to wind) & 2 & 0 \\
\hline Tweezers & 16 & 5 & Screwdriver & 1 & 1 \\
\hline Nail file & 15 & 6 & Cap (to unscrew) & 1 & 1 \\
\hline Shotgun & 14 & 4 & Needle & 1 & 1 \\
\hline Pocket calculator & 12 & 4 & Cork-screw & 1 & 0 \\
\hline Comb & 10 & 1 & Flashlight & 1 & 0 \\
\hline Wash glove & 9 & 3 & Wrist watch (to wind up) & 1 & 0 \\
\hline Match (to light) & 9 & 2 & Letter (to post a) & 1 & 0 \\
\hline Toothbrush & 8 & 5 & Bonnet (to put on) & 1 & 0 \\
\hline Razor & 8 & 5 & Book (to leaf through) & 1 & 0 \\
\hline Shoebrush & 8 & 3 & Drill & 1 & 0 \\
\hline Pliers (extract a nail) & 8 & 2 & Umbrella (to open) & 0 & 0 \\
\hline Fan & 8 & 2 & Tap (to open) & 0 & 0 \\
\hline Electric drill & 7 & 4 & Door (to open) & 0 & 0 \\
\hline Knife sharpener & 7 & 3 & Rope (to climb) & 0 & 0 \\
\hline Saw & 7 & 1 & Broom & 0 & 0 \\
\hline Whistle & 6 & 3 & Key & 0 & 0 \\
\hline TV remote control & 5 & 4 & Spoon (To eat soup with) & 0 & 0 \\
\hline Drum (to play) & 5 & 1 & Whiskbroom & 0 & 0 \\
\hline To butter a slice of bread & 5 & 1 & Coin (to flip) & 0 & 0 \\
\hline Knife & 5 & 0 & To squeeze an orange & 0 & 0 \\
\hline Fork & 4 & 2 & Marbles (to play) & 0 & 0 \\
\hline Pencil sharpener & 4 & 2 & Piano (to play) & 0 & 0 \\
\hline Magazine (to open) & 4 & 2 & Bottle-opener & 0 & 0 \\
\hline Lighter & 4 & 2 & Ball ( to throw) & 0 & 0 \\
\hline Banana (to peel) & 4 & 1 & Apple (to eat) & 0 & 0 \\
\hline Iron & 4 & 0 & Gloves (to put on) & 0 & 0 \\
\hline Balloon (to inflate) & 3 & 1 & Glasses (to put on) & 0 & 0 \\
\hline Water glass (to drink) & 3 & 0 & Necklace (to put on) & 0 & 0 \\
\hline Teaspoon (to mix) & 3 & 0 & Sieve (to put flour through) & 0 & 0 \\
\hline Ring (to put) & 3 & 0 & Cigarette (to smoke) & 0 & 0 \\
\hline Pipe (to smoke) & 2 & 1 & & & \\
\hline
\end{tabular}

